\title{
Complete ${ }^{1} \mathrm{H}$ - and ${ }^{13} \mathrm{C}$ - Resonance Assignment of Methyl
}

\section{$2 \alpha, 3 \beta, 24-T r i-O-a c e t y l u r s-12-e n-28-0 a t e ~ a n d ~ M e t h y l ~ 2 \alpha, 3 \beta$,}

\section{4-Tri-O-acetylolean-12-en-28-oate by NMR Spectroscopy}

\author{
F.J.Q. Monte ${ }^{*}$, and J.P. Kintzinger ${ }^{b}$ \\ ${ }^{a}$ Departamento de Química Orgânica e Inorgânica, Centro de Ciências, Universidade \\ Federal do Ceará, 60021-970 Fortaleza - CE, Brazil; \\ ${ }^{b}$ Laboratoire de RMN et de Modelisation Moléculaire, UA 422 du CNRS, Faculté de \\ Chimie, Université Louis Pasteur, 67008-Strasbourg, France
}

Received: August 1, 1997

\begin{abstract}
O trabalho descreve o estudo de dois triterpenos isômeros (1: $2 \alpha, 3 \beta, 24$-tri-O-acetil-12-eno-28ursolato de metila e 2: $2 \alpha, 3 \beta, 24$-tri-O-acetil-12-eno-28-oleanato de metila) efetuando a completa atribuição dos deslocamentos químicos dos hidrogênios e carbonos. Foram utilizadas técnicas de ressonância magnética nuclear $\left({ }^{1} \mathrm{H} \mathrm{e}{ }^{13} \mathrm{C}\right)$ uni- e bidimensionais empregando os seguintes passos: a) Análise comparativa dos espectros de RMN ${ }^{13} \mathrm{C}$-PND e RMN ${ }^{13} \mathrm{C}$-DEPT para identificação dos carbonos quaternários, metínicos, metilênicos, e metílicos; b) aplicação da técnica ${ }^{1} \mathrm{H} \mathrm{x}{ }^{13} \mathrm{C} \mathrm{HMBC}$ [acoplamento de higrogênio e carbono-13 via duas $\left({ }^{2} \mathrm{~J}_{\mathrm{CH}}\right)$ e três $\left({ }^{3} \mathrm{~J}_{\mathrm{CH}}\right)$ ligações] para atribuição dos deslocamentos químicos dos espectros de ${ }^{13} \mathrm{C}$; c) uso dos espectros ${ }^{1} \mathrm{H} \mathrm{x}{ }^{13} \mathrm{C}$ HMQC [interação spin-spin de higrogênio e carbono-13 via uma $\left({ }^{1} \mathrm{~J}_{\mathrm{CH}}\right)$ ligação] para determinar os deslocamentos químicos dos átomos de hidrogênio e para confirmar os dos carbonos hidrogenados; d) uso dos espectros bidimensionais de correlação homonuclear de hidrogênio e hidrogênio $\left({ }^{1} \mathrm{H} \mathrm{x}{ }^{1} \mathrm{H}\right.$-COSY), e de efeito nuclear Overhauser homonuclear de hidrogênio e hidrogênio ( ${ }^{1} \mathrm{H} x{ }^{1} \mathrm{H}$ - NOESY) para confirmar os sinais de hidrogênios e para determinações configuracionais $(\alpha$ e $\beta$ ) dos hidrogênios metilênicos e metínicos e, e) análise dos padrões de desdobramento (mutiplicidade e constante de acoplamento) nos espectros unidimensionais para confirmar os sinais de vários átomos de hidrogênio. Foram descritas as condições experimentais dos aparelhos utilizados (RMN $500 \mathrm{MHz}$, para hidrogênio e $125 \mathrm{MHz}$, para carbonos) e de isolamento dos constituintes $\mathbf{1}$ e $\mathbf{2}$. Todos os resultados são resumidos na forma de tabelas.
\end{abstract}

The complete ${ }^{1} \mathrm{H}$ and ${ }^{13} \mathrm{C}$ chemical shift assignments of extended hydrogen spin systems in triterpenoid derivatives (methyl $2 \alpha, 3 \beta, 24$-tri-O-acetylurs-12-en-28-oate and methyl $2 \alpha, 3 \beta, 24$-triO-acetylolean-12-en-28-oate) was accomplished making use of one and two dimensional NMR techniques (HMBC, HMQC, COSY and NOESY).

Keywords: triterpenoids, complete ${ }^{1} H$ and ${ }^{13} C$-NMR signal assignments; $1 D$ NMR; $2 D$ NMR $\left({ }^{1} \mathrm{Hx}{ }^{13} \mathrm{C}\right.$-HMBC, ${ }^{1} \mathrm{H} x{ }^{13} \mathrm{C}-\mathrm{HMQC},{ }^{1} \mathrm{H} x{ }^{1} \mathrm{H}$-COSY, ${ }^{1} \mathrm{H} x{ }^{1} \mathrm{H}$-NOESY $)$

\section{Introduction}

The extraordinary advances made in spectroscopic techniques have enormously accelerated the research in the field of isolation and structure elucidation of complex natural products. Of all the physical methods, the NMR technique has greatly changed during the last two decades mainly by introduction and development of multiple pulse and 2D NMR. Consequently, a large number of triterpenes have been examined by NMR spectroscopy and much chemical shift data has been accumulated and utilized in further investigations of these natural products. On the 
other hand, the use of these secondary metabolites as therapeutical agents has been the subject of extensive exploratory activities during recent years ${ }^{1}$. The ${ }^{1} \mathrm{H}$ and ${ }^{13} \mathrm{C}$ triterpene chemical shifts provided useful information concerning conformations and configurations of these complex organic derivatives and are also useful for the better understanding of the correlations between their molecular conformations and their biological activities ${ }^{2}$.

In this paper we report an extensive NMR study of two isomeric $\left(\mathrm{C}_{37} \mathrm{H}_{56} \mathrm{O}_{8}\right)$ triterpenoids derivatives with their complete ${ }^{1} \mathrm{H}$ and ${ }^{13} \mathrm{C}$ signal assignments, by application of $1 \mathrm{D}$ and 2D spectral experiments. The compounds investigated were methyl $2 \alpha, 3 \beta, 24$-tri-O-acetylurs-12-en-28-oate (1) and methyl $2 \alpha, 3 \beta, 24$-tri-O-acetylolean-12-en-28-oate (2) obtained after acetylation and methylation of a mixture isolated from Mentha villosa $\mathrm{Huds}^{3}$. This plant is used as a remedy in the treatment of amebiasis, giardiasis ${ }^{4}$ and shistosomiasis 5 .

This is the first report giving the complete assignment of these pentacyclic triterpenoid derivatives of ursolic and oleanolic acids. The following steps were concurrently employed: a) comparative analysis of the ${ }^{13} \mathrm{C}-\mathrm{NMR}-\mathrm{PND}$ and ${ }^{13} \mathrm{C}$-NMR-DEPT for identification of quaternary, methine, methylene and methyl carbon atoms; b) application of the HMBC experiment to chemical shift assignment of the ${ }^{13} \mathrm{C}$ spectra; $\mathrm{c}$ ) use of the HMQC spectra to determine the chemical shifts of the hydrogen atoms and to confirm those of the hydrogenated carbons; d) use of hydrogen ${ }^{1} \mathrm{H}$ $\mathrm{x}^{1} \mathrm{H}$ - COSY and ${ }^{1} \mathrm{H} \mathrm{x}{ }^{1} \mathrm{H}$ - NOESY maps to confirm the ${ }^{1} \mathrm{H}$ assignments (and, indirectly, also the ${ }^{13} \mathrm{C}$ assignments) and to establish the configurational assignment ( $\alpha$ and $\beta$ ) of all methylene and methine hydrogens and e) analysis of the splitting patterns (multiplicity and coupling constant) in the 1D NMR spectra to confirm the resonances (including the configurational assignment) of various hydrogen atoms.

\section{Experimental}

\section{Plant material}

Mentha villosa was collected in the "Horto de Plantas Medicinais" of the "Universidade Federal do Ceará", Fortaleza, Brazil. A voucher of the plant (N. 16.545) is deposited in the Herbarium "Prisco Bezerra" of the Departamento de Biologia of the Universidade Federal do Ceará.

\section{Isolation procedure}

The fraction obtained from the ETOH extract by partition with $\mathrm{CHCl}_{3}$ was successively chromatographed on silica gel column to afford fraction $\mathrm{E}$ (eluted with hexane$\mathrm{CHCl}_{3}$ 2:8). Fraction E was methylated with $\mathrm{CH}_{2} \mathrm{~N}_{2}$ and then acetylated with $\mathrm{Ac}_{2} \mathrm{O}$ /pyridine in the usual manner to yield a product named E-MeAc. Silica gel preparative TLC of E-MeAc lead to a fraction $\left(\mathrm{R}_{\mathrm{f}} 0.60\right.$, eluted with $\left.\mathrm{CH}_{2} \mathrm{Cl}_{2}\right)$,

Table 1. NMR data for methyl $2 \alpha, 3 \beta, 24$-tri-O-acetylurs-12-en-28-oate (1)*.

\begin{tabular}{|c|c|c|c|c|c|}
\hline \multirow[b]{2}{*}{ C } & \multicolumn{2}{|r|}{${ }^{1} \mathrm{H} \mathrm{x}^{13} \mathrm{C}-\mathrm{HMQC}\left({ }^{1} \mathrm{~J}_{\mathrm{CH}}\right)$} & \multicolumn{2}{|c|}{${ }^{1} \mathrm{H} \mathrm{x}^{13} \mathrm{C}-\mathrm{HMBC}$} & \multirow[t]{2}{*}{${ }^{1} \mathrm{H} \mathrm{x}^{1} \mathrm{H}-\mathrm{NOESY}$} \\
\hline & $\delta_{\mathrm{C}}$ & $\delta_{\mathrm{H}}$ & ${ }^{2} \mathrm{~J}_{\mathrm{CH}}$ & ${ }^{3} \mathrm{~J}_{\mathrm{CH}}$ & \\
\hline 4 & 43.00 & --- & $3 \mathrm{H}-23,2 \mathrm{H}-24$ & & --- \\
\hline 8 & 39.30 & --- & $3 \mathrm{H}-26$ & $3 \mathrm{H}-27$ & --- \\
\hline 10 & 37.50 & --- & $3 \mathrm{H}-25$ & & --- \\
\hline 13 & 138.60 & --- & & $3 \mathrm{H}-27$ & --- \\
\hline 14 & 41.80 & --- & $3 \mathrm{H}-27$ & $3 \mathrm{H}-26$ & --- \\
\hline 17 & 48.00 & --- & & & --- \\
\hline 28 & 178.00 & --- & & $\mathrm{MeO}-28$ & --- \\
\hline $\mathrm{AcO}-2$ & 170.80 & --- & $\underline{\mathrm{H}}_{3} \mathrm{CCO}_{2}-2$ & $\mathrm{H}-2$ & --- \\
\hline $\mathrm{AcO}-3$ & 171.00 & --- & $\underline{\mathrm{H}}_{3} \mathrm{CCO}_{2}-3$ & $\mathrm{H}-3$ & --- \\
\hline AcO-24 & 171.10 & --- & & $2 \mathrm{H}-24$ & --- \\
\hline \multicolumn{6}{|l|}{ CH } \\
\hline 2 & 69.28 & $5.16(\mathrm{dt}, \mathrm{J}=12.0,4.8 \mathrm{~Hz}, \mathrm{H}-2 \beta)$ & & & $\mathrm{H}-1 \beta, 2 \mathrm{H}-24,3 \mathrm{H}-25$ \\
\hline 3 & 79.90 & $4.82(\mathrm{~d}, \mathrm{~J}=12.0 \mathrm{~Hz}, \mathrm{H}-3 \alpha)$ & & $3 \mathrm{H}-23,2 \mathrm{H}-24$ & $\mathrm{H}-1 \alpha, \mathrm{H}-5 \alpha, 3 \mathrm{H}-23$ \\
\hline 5 & 55.52 & $1.07(\mathrm{H}-5 \alpha)$ & & $2 \mathrm{H}-24,3 \mathrm{H}-25$ & $\mathrm{H}-3 \alpha, \mathrm{H}-7 \alpha, \mathrm{H}-9 \alpha$ \\
\hline 9 & 47.60 & $1.57(H-9 \alpha)$ & & $3 \mathrm{H}-25,3 \mathrm{H}-26$ & H-1 $\alpha, \mathrm{H}-5 \alpha, 3 \mathrm{H}-27$ \\
\hline 12 & 125.00 & $5.24(\mathrm{t}, \mathrm{J}=3.5 \mathrm{~Hz})$ & & & $2 \mathrm{H}-11, \mathrm{H}-18 \beta$ \\
\hline
\end{tabular}


Table 1. (cont.)

\begin{tabular}{llcccc}
\hline 18 & 52.85 & $2.24(\mathrm{~d}, \mathrm{~J}=11.4 \mathrm{~Hz}, \mathrm{H}-18 \beta)$ & & $3 \mathrm{H}-29$ & H-12, H-22ß, 3H-29 \\
19 & 39.10 & $1.32(\mathrm{H}-19 \alpha)$ & $3 \mathrm{H}-29$ & $3 \mathrm{H}-30$ & $3 \mathrm{H}-27,3 \mathrm{H}-29,3 \mathrm{H}-30$ \\
20 & 38.87 & $0.98(\mathrm{H}-20 \beta)$ & $3 \mathrm{H}-30$ & $3 \mathrm{H}-29$ & $3 \mathrm{H}-29,3 \mathrm{H}-30$
\end{tabular}

\section{$\mathrm{CH}_{2}$}

1

6

7

11

15

16

21

22

24

\section{$\mathrm{CH}_{3}$}

23

25

26

27

29

30

AcO-2

AcO-3

AcO-24

MeO-28
44.32

$2.11(\mathrm{dd}, \mathrm{J}=12.4,4.8 \mathrm{~Hz}, \mathrm{H}-1 \beta)$

$$
1.10(\mathrm{t}, \mathrm{J}=12.4 \mathrm{~Hz}, \mathrm{H}-1 \alpha)
$$

19.20

32.20

23.30

27.90

24.20

30.65

36.60

65.50

$1.58(\mathrm{dt}, \mathrm{J}=10.4,4.2 \mathrm{~Hz}, \mathrm{H}-22 \beta)$

$1.64(\mathrm{H}-6 \alpha)$

$1.47(\mathrm{H}-6 \beta)$

$1.48(\mathrm{H}-7 \alpha)$

$1.37(\mathrm{H}-7 \beta)$

1.92 (m, H-11 $\alpha, \mathrm{H}-11 \beta)$

$$
1.76(\mathrm{dt}, \mathrm{J}=14.0,4.9 \mathrm{~Hz}, \mathrm{H}-15 \beta)
$$$$
1.04(\mathrm{H}-15 \alpha)
$$

$2.02(\mathrm{dt}, \mathrm{J}=12.4,4.9 \mathrm{~Hz}, \mathrm{H}-16 \alpha)$

$1.67(\mathrm{H}-16 \beta)$

$1.47(\mathrm{H}-21 \beta)$

$1.30(\mathrm{H}-21 \alpha)$

$$
1.66(\mathrm{H}-22 \alpha)
$$

4.20 (s, 2H-84)

$3 \mathrm{H}-25$

H- $1 \alpha$, H-2 $\beta, 3 \mathrm{H}-25$

H-1 $\beta, H-3 \alpha, H-9 \alpha$

Н-6 $\beta$, H-7 $\alpha, 3 \mathrm{H}-23$

$\mathrm{H}-6 \alpha$

$3 \mathrm{H}-26$

$3 \mathrm{H}-27$
$\mathrm{H}-15 \beta$
H-16 $\beta$, H-19 $\alpha$, H-22 $\alpha$

$$
\mathrm{H}-16 \alpha
$$

-15 $\alpha, 3 \mathrm{H}-26$

$3 \mathrm{H}-30$

$\mathrm{H}-21 \alpha, \mathrm{H}-22 \beta$

H-16 $\alpha, \mathrm{H}-21 \beta, 3 \mathrm{H}-30$

H-16 $\alpha, \mathrm{H}-22 \beta$

H-18, H-21 $\beta, \mathrm{H}-22 \alpha$

$3 \mathrm{H}-23$

H-2 $\beta, 3 \mathrm{H}-23,3 \mathrm{H}-25$

$2 \mathrm{H}-24$

$\mathrm{H}-3 \alpha, 2 \mathrm{H}-24$

H-6 $\beta, 2 \mathrm{H}-11,2 \mathrm{H}-24,3 \mathrm{H}-26$

Н-6 $\beta$, H-7 $\beta, 2 \mathrm{H}-11, \mathrm{H}-$ $15 \beta, 3 \mathrm{H}-25$

H-7 $\alpha, \mathrm{H}-9 \alpha$

$\mathrm{H}-18 \beta, \mathrm{H}-19 \alpha, \mathrm{H}-20 \beta$

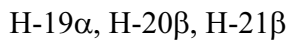

* Multiplicity of signals of carbon atoms deduced by comparative analysis of PND- and DEPT- ${ }^{13} \mathrm{C}-\mathrm{NMR}$. Chemical shifts of hydrogen atoms obtained from $1 \mathrm{D}{ }^{1} \mathrm{H}-\mathrm{NMR}$. The $2 \mathrm{D}^{1} \mathrm{H} \mathrm{x}{ }^{1} \mathrm{H}-\mathrm{COSY}$ and $2 \mathrm{D}{ }^{1} \mathrm{H} \mathrm{x}{ }^{13} \mathrm{C}$ - HMQC spectra were also used in these assignments.

identified as a mixture of the triterpene derivatives $(\mathbf{1}$ and 2) which were separated by preparative HPLC Waters model 6000A, detector R - 401 diferential refractometer; RP 18 (250 x $9.4 \mathrm{~mm})$ column; mobile phase: methanolwater $98: 2$, flow-rate of $2 \mathrm{~mL} \mathrm{~min}^{-1}$.

NMR spectra

${ }^{1} \mathrm{H}$ - and ${ }^{13} \mathrm{C}-\mathrm{NMR}$ experiments were performed on a BRUKER ARX 500 spectrometer working at $500.1 \mathrm{MHz}$ for hydrogen and $125.75 \mathrm{MHz}$ for ${ }^{13} \mathrm{C}$ carbon, using $\mathrm{CDCl}_{3}$ as solvent. Solutions were made from $0.35 \mathrm{ml}$ of $\mathrm{CDCl}_{3}$ and 2-8 mg of triterpenes with TMS as the internal standard. For all experiments the temperature was stabilised at 298 K. For the NOESY experiments, the samples were degased by bubbling nitrogen through the solution and fitting a teflon serum cap. The 2D experiments were acquired and processed with the software provided by BRUKER on ASPECT X32. 
Typical acquisition and processing conditions for COSY and NOESY experiments were: relaxation delay of 1 to 2 seconds, 512 to $1024 t_{1}$ increments; 1024 to $2048 t_{2}$ points; sweep width of $6 \mathrm{ppm}$. Sine bell squared and shifted $(\pi / 4, \pi / 6$ and $\pi / 8)$ apodization functions were used for processing. The mixing time in the NOESY experiments, generally set at 1.2-1.5 seconds, was also varied between 0.8 and 2 seconds, without substantial change in the results. For ${ }^{1} \mathrm{H} \mathrm{x}{ }^{13} \mathrm{C}\left({ }^{13} \mathrm{C}\right.$ detected $)$ and ${ }^{13} \mathrm{C} \mathrm{x}{ }^{1} \mathrm{H}\left({ }^{1} \mathrm{H}\right.$ detected $)$ correlations, the same relaxation delay was used, 512 to $1024 t_{1}$ increments, 1024 to $2048 t_{2}$ points, the sweep width being respectively $7 \mathrm{ppm}$ for ${ }^{1} \mathrm{H}$ and $180 \mathrm{ppm}$ for ${ }^{13} \mathrm{C}$. Lorentzian and Gaussian deconvolution were generally used in the processing. The number of scans was set for an overall acquisition time of about $12 \mathrm{~h}$ to $16 \mathrm{~h}$.

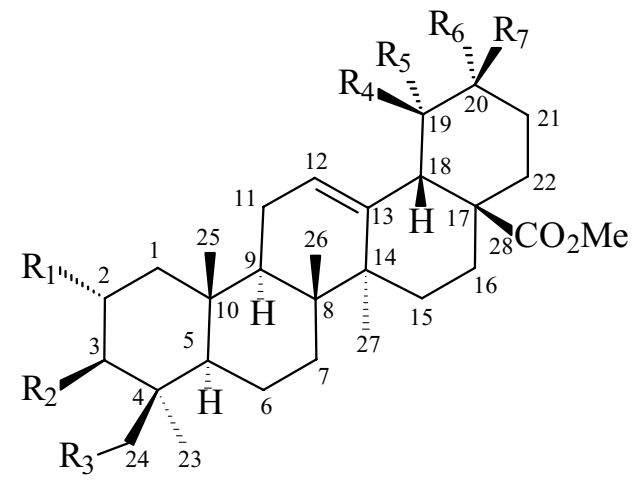

$\begin{array}{lccccccc} & \mathrm{R}_{1} & \mathrm{R}_{2} & \mathrm{R}_{3} & \mathrm{R}_{4} & \mathrm{R}_{5} & \mathrm{R}_{6} & \mathrm{R}_{7} \\ \mathbf{1} & \text { OAc } & \text { OAc } & \text { OAc } & \text { Me } & \mathrm{H} & \mathrm{Me} & \mathrm{H} \\ \mathbf{2} & \text { OAc } & \text { OAc } & \text { OAc } & \mathrm{H} & \mathrm{H} & \mathrm{Me} & \mathrm{Me} \\ \mathbf{3} & \text { OAc } & \text { OAc } & \text { H } & \mathrm{H} & \mathrm{H} & \mathrm{Me} & \mathrm{Me}\end{array}$

Figure 1. Structures of $2 \alpha, 3 \beta, 24$-tri-O-acetylurs-12-en-28-oate (1), $2 \alpha, 3 \beta, 24$-tri-O-acetylolean-12-en-28-oate (2) and $2 \alpha, 3 \beta$-di-O-acetylolean-12-en-28-oate (3).

\section{Results and Discussion}

An essential prerequisite to the unambiguous assignment of ${ }^{1} \mathrm{H}$ chemical shifts from ${ }^{13} \mathrm{C}-{ }^{1} \mathrm{H}$ shift correlated spectra is to first unambiguously assign the ${ }^{13} \mathrm{C}$ chemical shifts of protonated carbons ${ }^{6,7}$.

The signals corresponding to quaternary, methine, methylene and methyl carbon atoms were identified by comparative analysis of the ${ }^{13} \mathrm{C}-\mathrm{NMR}$ - PND and ${ }^{13} \mathrm{C}$ NMR DEPT spectra. The ${ }^{1} \mathrm{H} \mathrm{x}{ }^{13} \mathrm{C}$ - HMBC Heteronuclear Multiple Connectivity - coupling of hydrogen and carbon13 via two $\left({ }^{2} \mathrm{~J}_{\mathrm{CH}}\right)$ and three $\left({ }^{3} \mathrm{~J}_{\mathrm{CH}}\right)$ bonds spectra were successfully used to attribute the chemical shifts of several protonated as well as, of almost all non-protonated carbons. For example, the singlet at $\delta_{\mathrm{H}} 4.20$ in the ${ }^{1} \mathrm{H}-\mathrm{NMR}$ spectrum of 1 (Fig. 1) was correlated with hydrogens attached at an oxygenated carbon. Assuming this resonance assignable to $2 \mathrm{H}-24$, the application of the HMBC technique led to the assignment of $\mathrm{CH}-3, \mathrm{C}-4, \mathrm{CH}-5$ and $\mathrm{CH}_{3}-23$ by identifying ${ }^{2} \mathrm{~J}_{\mathrm{CH}}$ and ${ }^{3} \mathrm{~J}_{\mathrm{CH}}$ connectivities. Thus, the carbon atom C-4 (C 43.00 ) is readily assigned as the only quaternary carbon of the four; $\mathrm{C}-3\left(\delta_{\mathrm{C}} 79.90\right)$ is an oxygenated carbon and C-5 $\left(\delta_{\mathrm{C}} 55.52\right)$ is distinguished by additional $3 \mathrm{H}-25\left(\delta_{\mathrm{H}} 1.07\right)$ cross peak $\left({ }^{3} \mathrm{~J}_{\mathrm{CH}}\right)$ for the latter carbon, while $\mathrm{CH}_{3}-23\left(\delta_{\mathrm{CH}}\right.$ 23.10) was identified as a methyl carbon. Working along the molecule in this fashion, using the hydrogens from only the six methyl resonances, allowed in addition unambiguous assignment of $\mathrm{CH}_{2}-1\left(\delta_{\mathrm{C}} 44.32\right), \mathrm{CH}_{2}-7\left(\delta_{\mathrm{C}} 33.20\right), \mathrm{C}-8$ $\left(\delta_{\mathrm{C}} 39.30\right), \mathrm{CH}-9\left(\delta_{\mathrm{C}} 47.60\right), \mathrm{C}-10\left(\delta_{\mathrm{C}} 37.50\right), \mathrm{C}-13\left(\delta_{\mathrm{C}}\right.$ 138.60), C-14 ( $\left.\delta_{\mathrm{C}} 41.80\right), \mathrm{CH}_{2}-15\left(\delta_{\mathrm{C}} 27.90\right), \mathrm{CH}-18\left(\delta_{\mathrm{C}}\right.$ $52.85), \mathrm{CH}-19\left(\delta_{\mathrm{C}} 39.10\right), \mathrm{CH}-20\left(\delta_{\mathrm{C}} 38.87\right), \mathrm{CH}_{2}-21\left(\delta_{\mathrm{C}}\right.$ $30.65)$ and $\mathrm{CH}_{2}-24\left(\delta_{\mathrm{C}} 65.50\right)$ (Table 1$)$. The quaternary carbons $\mathrm{C}-8\left(\delta_{\mathrm{C}} 39.30\right)$ and $\mathrm{C}-14\left(\delta_{\mathrm{C}} 41.80\right)$ of 1 were distinguished from the earlier assignment of $\mathbf{3}^{8}$ (Fig. 1) since the chemical shifts of these carbon atoms are almost invariant for $\mathbf{1}$ and $\mathbf{3}$. Thus, in the HMBC spectrum of $\mathbf{3}$, only the carbon C-8 $\left(\delta_{\mathrm{C}} 39.35\right)$ showed connectivities with the hydrogen atoms $2 \mathrm{H}-11\left(\delta_{\mathrm{H}} 1.90-1.85\right)$, while only the

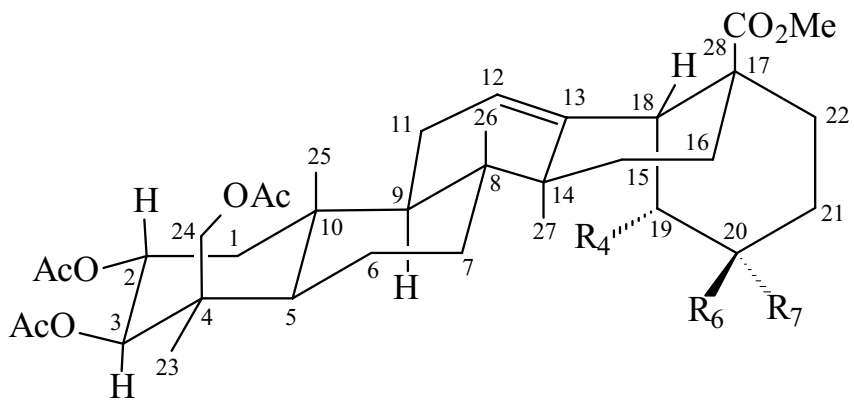

$$
\begin{array}{llll}
\text { 1a } & \mathrm{R}_{4}=\mathrm{Me} & \mathrm{R}_{6}=\mathrm{Me} & \mathrm{R}_{7}=\mathrm{H} \\
\text { 2a } & \mathrm{R}_{4}=\mathrm{H} & \mathrm{R}_{6}=\mathrm{Me} & \mathrm{R}_{7}=\mathrm{Me}
\end{array}
$$

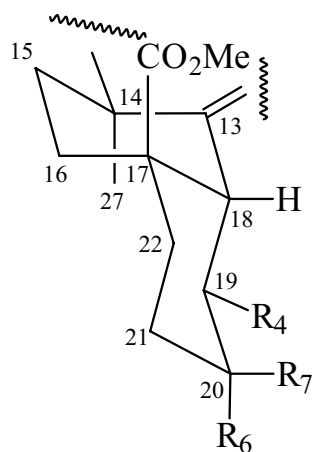

4

Figure 2. Stereochemical view of the structures of 1 and 2. 
carbon C-14 $\left(\delta_{\mathrm{C}} 41.69\right)$ showed a correlation with the hydrogen atom $\mathrm{H}-12\left(\delta_{\mathrm{H}} 5.26\right)$. On the other hand, the $\mathrm{H}-18$ doublet $\left(\delta_{\mathrm{H}} 2.24, \mathrm{~J}=11.4 \mathrm{~Hz}\right)$ of 1 allowed the localization of $\mathrm{H}-19\left(\delta_{\mathrm{H}} 1.32\right)$ in the ${ }^{1} \mathrm{H} \mathrm{x}{ }^{1} \mathrm{H}$ - COSY spectrum which in turn determined the chemical shift of the $\mathrm{CH}-19\left(\delta_{\mathrm{H}}\right.$ 39.35) through ${ }^{1} \mathrm{H}-{ }^{13} \mathrm{C}$ - HMQC Heteronuclear Multiple Quantum Coherence - spin-spin interaction of hydrogen and carbon-13 via one $\left({ }^{1} \mathrm{~J}_{\mathrm{CH}}\right)$ bond spectrum. Thus, the resonance at $\delta_{\mathrm{C}} 38.87$ correlates to $\mathrm{CH}-20$. Two methine (CH-2 and CH-12), four methylene $\left(\mathrm{CH}_{2}-6, \mathrm{CH}_{2}-11, \mathrm{CH}_{2}-\right.$ 16 and $\left.\mathrm{CH}_{2}-22\right)$ and one quaternary $(\mathrm{C}-17)$ carbons showed no cross peak with methyl hydrogens in the HMBC experiment as expected. Nevertheless, all were unambiguously identified. $\mathrm{CH}-2\left(\delta_{\mathrm{C}} 69.28\right)$ is readily assigned as an oxygenated carbon and $\mathrm{CH}-12\left(\delta_{\mathrm{C}} 125.00\right)$ is a sp ${ }^{2}$ carbon. The methylene carbons were identified by use of a COSY technique. For example, the signal of $\mathrm{H}-9\left(\delta_{\mathrm{H}} 1.57\right)$ allowed the localization of the $2 \mathrm{H}-11\left(\delta_{\mathrm{H}} 1.92\right)$ hydrogens which in turn, lead to assignment of $\mathrm{CH}_{2}-11\left(\delta_{\mathrm{C}} 23.30\right)$ from the HMQC spectrum. In this way, were also assigned the chemical shifts of the $\mathrm{CH}_{2}-16\left(\delta_{\mathrm{C}} 24.20\right)$ and $\mathrm{CH}_{2}-22\left(\delta_{\mathrm{C}}\right.$ 36.60) carbons. The overcrowded region of the COSY map did not allow the characterization of the $2 \mathrm{H}-6$ hydrogens with respect to $2 \mathrm{H}-7$. By exclusion, the last signal correspondening to one methylene carbon was attributed to $\mathrm{CH}_{2}-6\left(\delta_{\mathrm{C}} 19.20\right)$. The remaining quaternary carbon $\mathrm{C}-17$ $\left(\delta_{\mathrm{C}} 48.00\right)$ was identified by comparative analysis of ${ }^{13} \mathrm{C}$ NMR - PND and ${ }^{13} \mathrm{C}-\mathrm{NMR}$ - DEPT spectra.

Finally, all methyl ${ }^{13} \mathrm{C}$ signals were assigned from their connectivities with assigned methyl ${ }^{1} \mathrm{H}$ signals. These methyl ${ }^{1} \mathrm{H}$ signals were identified and distinguished on the basis of the observed connectivities in the HMBC map with

Table 2. NMR data for methyl $2 \alpha, 3 \beta, 24$-tri-O-acetylolean-12-en-28-oate (2)*.

\begin{tabular}{|c|c|c|c|c|c|}
\hline \multirow[b]{2}{*}{$\mathbf{C}$} & \multicolumn{2}{|r|}{${ }^{1} \mathrm{H} \mathrm{x}^{13} \mathrm{C}-\mathrm{HMQC}\left({ }^{1} \mathrm{~J}_{\mathrm{CH}}\right)$} & \multicolumn{2}{|c|}{${ }^{1} \mathrm{H} x{ }^{13} \mathrm{C}-\mathrm{HMBC}$} & \multirow[t]{2}{*}{${ }^{1} \mathrm{H} x^{1} \mathrm{H}-\mathrm{NOESY}$} \\
\hline & $\delta_{C}$ & $\delta_{\mathrm{H}}$ & ${ }^{2} J_{\mathrm{CH}}$ & ${ }^{3} \mathrm{~J}_{\mathrm{CH}}$ & \\
\hline 4 & 43.40 & --- & $\mathrm{H}-3,3-\mathrm{H} 23,2 \mathrm{H}-2$ & & --- \\
\hline 8 & 39.70 & --- & $3 \mathrm{H}-25$ & & --- \\
\hline 10 & 38.50 & --- & $\mathrm{H}-1 \beta$ & & --- \\
\hline 13 & 143.90 & --- & & $H-15 \beta$ & --- \\
\hline 14 & 42.20 & --- & $\mathrm{H}-15 \beta$ & & --- \\
\hline 17 & 47.30 & --- & & & --- \\
\hline 20 & 30.80 & --- & $3 \mathrm{H}-29,3 \mathrm{H} 30$ & & --- \\
\hline 28 & 178.40 & --- & & $\mathrm{MeO}-28$ & --- \\
\hline $\mathrm{AcO}-2$ & 170.80 & --- & $\underline{\mathrm{H}}_{3} \mathrm{CCO}_{2}-2$ & $\mathrm{H}-2$ & --- \\
\hline $\mathrm{AcO}-3$ & 171.00 & --- & $\underline{\mathrm{H}}_{3} \mathrm{CCO}_{2}-3$ & $\mathrm{H}-3$ & --- \\
\hline $\mathrm{AcO}-24$ & 171.10 & --- & $\underline{\mathrm{H}}_{3} \mathrm{CCO}_{2}-24$ & $2 \mathrm{H}-24$ & --- \\
\hline \multicolumn{6}{|l|}{ CH } \\
\hline 2 & 69.26 & $5.15(\mathrm{dt}, \mathrm{J}=12.0,4.8 \mathrm{~Hz}, \mathrm{H}-2 \beta)$ & $\mathrm{H}-1 \beta, \mathrm{H}-3$ & & $\mathrm{H}-1 \beta, 2 \mathrm{H}-24,3 \mathrm{H}-25$ \\
\hline 3 & 79.87 & $4.82(\mathrm{~d}, \mathrm{~J}=12.0 \mathrm{~Hz}, \mathrm{H}-3 \alpha)$ & & $\mathrm{H}-1 \beta, 2 \mathrm{H}-24$ & $H-1 \alpha, H-5 \alpha, 3 H-23$ \\
\hline 5 & 55.52 & $1.08(\mathrm{H}-5 \alpha)$ & & $\begin{array}{l}\mathrm{H}-1 \beta, 2 \mathrm{H}- \\
24,3 \mathrm{H}-25\end{array}$ & $H-3 \alpha$ \\
\hline 9 & 47.74 & $1.58(H-9 \alpha)$ & & $3 \mathrm{H}-25,3 \mathrm{H}-26$ & $3 \mathrm{H}-27$ \\
\hline 12 & 121.84 & $5.27(\mathrm{t}, \mathrm{J}=4.0 \mathrm{~Hz})$ & & & $2 \mathrm{H}-11, \mathrm{H}-18 \beta$ \\
\hline 18 & 41.26 & $2.86(\mathrm{dd}, \mathrm{J}=12.0,3.4 \mathrm{~Hz}, \mathrm{H}-18 \beta)$ & & & $\mathrm{H}-12,3 \mathrm{H}-30$ \\
\hline \multicolumn{6}{|l|}{$\mathrm{CH}_{2}$} \\
\hline 1 & 44.10 & $\begin{array}{l}2.08(\mathrm{H}-1 \beta) \\
1.07(\mathrm{H}-1 \alpha)\end{array}$ & & $3 \mathrm{H}-25$ & $\begin{array}{c}\mathrm{H}-2 \beta, 3 \mathrm{H}-25 \\
\mathrm{H}-3 \alpha\end{array}$ \\
\hline 6 & 19.32 & $\begin{array}{l}1.64(\mathrm{H}-6 \alpha) \\
1.47(\mathrm{H}-6 \beta)\end{array}$ & & & $\begin{array}{c}\mathrm{H}-5 \alpha \\
3 \mathrm{H}-26\end{array}$ \\
\hline
\end{tabular}


Table 2. (cont.)

\begin{tabular}{|c|c|c|c|c|c|}
\hline 7 & 32.90 & $\begin{array}{c}1.44(\mathrm{H}-7 \alpha)^{* *} \\
1.32(\mathrm{H}-7 \beta)\end{array}$ & & $3 \mathrm{H}-26$ & $\begin{array}{l}3 \mathrm{H}-27 \\
3 \mathrm{H}-26\end{array}$ \\
\hline 11 & 23.09 & $1.90(\mathrm{H}-11 \alpha, \mathrm{H}-11 \beta)$ & & & $\mathrm{H}-12,3 \mathrm{H}-25,3 \mathrm{H}-26$ \\
\hline 15 & 27.63 & $\begin{array}{l}1.62(\mathrm{H}-15 \beta) \\
1.07(\mathrm{H}-15 \alpha)\end{array}$ & & $3 \mathrm{H}-27$ & $\begin{array}{l}3 \mathrm{H}-26 \\
3 \mathrm{H}-27\end{array}$ \\
\hline 16 & 23.48 & $\begin{array}{l}1.97(\mathrm{H}-16 \alpha) \\
1.62(\mathrm{H}-16 \beta)\end{array}$ & H- $15 \beta$ & & $\begin{array}{c}\mathrm{H}-16 \beta, 3 \mathrm{H}-27 \\
\mathrm{H}-16 \alpha\end{array}$ \\
\hline 19 & 45.86 & $\begin{array}{c}1.64(\mathrm{H}-19 \alpha)^{* *} \\
1.13(\mathrm{H}-19 \beta)\end{array}$ & & $\begin{array}{c}\mathrm{H}-21 \beta, 3 \mathrm{H}- \\
29,3 \mathrm{H}-30\end{array}$ & $3 \mathrm{H}-30$ \\
\hline 21 & 33.87 & $\begin{array}{c}1.33(\mathrm{H}-21 \alpha)^{* *} \\
1.18(\mathrm{H}-21 \beta)\end{array}$ & & $3 \mathrm{H}-29,3 \mathrm{H}-30$ & \\
\hline 22 & 32.30 & $\begin{array}{l}1.68(\mathrm{H}-22 \beta) \\
1.57(\mathrm{H}-22 \alpha)\end{array}$ & & & $3 \mathrm{H}-30$ \\
\hline 24 & 65.44 & $4.20(\mathrm{~s})$ & & $\mathrm{H}-3,3 \mathrm{H}-23$ & $3 \mathrm{H}-23$ \\
\hline \multicolumn{6}{|l|}{$\mathrm{CH}_{3}$} \\
\hline 23 & 23.09 & $1.02(\mathrm{~s})$ & & $\mathrm{H}-3,2 \mathrm{H}-24$ & $\mathrm{H}-3 \alpha$ \\
\hline 25 & 16.18 & $1.05(\mathrm{~s})$ & & H-5 & $\mathrm{H}-1 \beta, \mathrm{H}-2 \beta, 2 \mathrm{H}-11$ \\
\hline 26 & 16.63 & $0.72(\mathrm{~s})$ & & & Н-6 $\beta$, H-7 $\beta, 2 \mathrm{H}-11, \mathrm{H}-15 \beta$ \\
\hline 27 & 25.83 & $1.11(\mathrm{~s})$ & & & H-7 $\alpha$, H-9 $\alpha$, H-15 $\alpha$, H-16 $\alpha$ \\
\hline 29 & 33.14 & $0.89(\mathrm{~s})$ & & $3 \mathrm{H}-30$ & \\
\hline 30 & 23.65 & $0.92(\mathrm{~s})$ & & $3 \mathrm{H}-29$ & 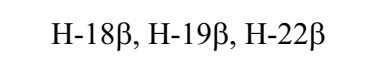 \\
\hline AcO-2 & 21.07 & $1.96(\mathrm{~s})$ & & & \\
\hline AcO-3 & $20.85 / 21.07$ & $2.04 / 2.05(\mathrm{~s})$ & & & \\
\hline $\mathrm{AcO}-24$ & $20.85 / 21.07$ & $2.04 / 2.05(\mathrm{~s})$ & & & \\
\hline $\mathrm{MeO}-28$ & 51.60 & $3.61(\mathrm{~s})$ & & & \\
\hline
\end{tabular}

* Multiplicity of signals of carbon atoms deduced by comparative analysis of PND- and DEPT- ${ }^{13} \mathrm{C}-\mathrm{NMR}$. Chemical shifts of hydrogen atoms obtained from $1 \mathrm{D}{ }^{1} \mathrm{H}-\mathrm{NMR}$. The $2 \mathrm{D}^{1} \mathrm{H} \mathrm{x}{ }^{1} \mathrm{H}-\mathrm{COSY}$ and $2 \mathrm{D}^{1} \mathrm{H} \mathrm{x}{ }^{13} \mathrm{C}$ - HMQC spectra were also used in these assignments.

** The H-7 and H-19/H-21 showed long range coupling ( $(\mathrm{J})$ with $3 \mathrm{H}-26$ and $3 \mathrm{H}-30$, respectively.

previously assigned carbon atoms (Table 1 ). In this way, only the signal of $3 \mathrm{H}-23\left(\delta_{\mathrm{H}} 1.03\right)$ showed a cross peak at the ${ }^{13} \mathrm{C}$ frequency with the readily assigned $\mathrm{CH}-3\left(\delta_{\mathrm{C}}\right.$ 79.90); $3 \mathrm{H}-25\left(\delta_{\mathrm{H}} 1.07\right)$ with $\mathrm{CH}_{2}-1\left(\delta_{\mathrm{C}} 44.32\right) ; 3 \mathrm{H}-26\left(\delta_{\mathrm{H}}\right.$ $0.74)$ with $\mathrm{CH}_{2}-7\left(\delta_{\mathrm{C}} 33.20\right) ; 3 \mathrm{H}-27\left(\delta_{\mathrm{H}} 1.06\right)$ with $\mathrm{C}-13$ $\left(\delta_{\mathrm{C}} 138.60\right) ; 3 \mathrm{H}-29\left(\delta_{\mathrm{H}} 0.84\right)$ with $\mathrm{CH}-18\left(\delta_{\mathrm{C}} 52.85\right)$ and $3 \mathrm{H}-30\left(\delta_{\mathrm{H}} 0.94\right)$ with $\mathrm{CH}_{2}-21\left(\delta_{\mathrm{C}} 30.65\right)$. The assignment of the carbon signals were then carried out by an HMQC experiment (Table 1).

The assignment of $\mathrm{CH}_{2}$ hydrogens as $\alpha$ or $\beta$ were obtained by analysis of the splitting patterns (multiplicity and coupling constant) in the 1D NMR, long range coupling ${ }^{9}\left({ }^{4} \mathrm{~J}\right)$ in the ${ }^{1} \mathrm{H} \times{ }^{1} \mathrm{H}-\mathrm{COSY}$ and especially ${ }^{1} \mathrm{H} \mathrm{x}$ ${ }^{1}$ H-NOESY spectra (Table 1). These spectra also indicated that $\mathbf{1}$ was in conformation 1a and confirmed the cis junction of rings $\mathrm{D}$ and $\mathrm{E}$ (see 4).

In a report of 3 $\beta$-O-acetyl-27-norurs-13-en-28-oic acid ${ }^{10}$, the chemical shifts of the carbon atoms $\mathrm{CH}_{3}-29\left(\delta_{\mathrm{C}}\right.$ 20.02) and $\mathrm{CH}_{3}-30\left(\delta_{\mathrm{C}} 17.66\right)$ were inconsistent with that of the same carbon atoms in compound $\mathbf{1}$. In the case of $\mathbf{1}$ the ${ }^{1} \mathrm{H} \mathrm{x}{ }^{13} \mathrm{C}$ - $\mathrm{HMBC}$ spectrum allowed the obvious assignment of $\mathrm{CH}_{3}-29\left(\delta_{\mathrm{C}} 16.95\right)$ by connectivities of the resonance at $\delta_{\mathrm{H}} 2.24(\mathrm{H}-18)$ to the resonance at $\delta_{\mathrm{C}} 16.95\left(\mathrm{CH}_{3}-29\right)$ and of the resonance at $\delta_{\mathrm{C}} 52.85(\mathrm{CH}-18)$ to the resonance at $\delta_{\mathrm{H}} 0.84(3 \mathrm{H}-29)$. Consequently, the signal at $\delta_{\mathrm{C}} 21.15$ was attributed to $\mathrm{CH}_{3}-30$.

Using the same procedure, the HMBC, COSY, NOESY and HMQC spectra furnished the ${ }^{1} \mathrm{H}$ and ${ }^{13} \mathrm{C}$ chemical 
shifts of compound 2 (Fig. 1, Table 2); and also the molecular conformation 2a and cis ring jucntion as in $\mathbf{4}$.

\section{Acknowledgments}

The authors gratefully acknowledge Coordenação de Aperfeiçoamento de Pessoal de Nível Superior (CAPES), Brazilian Agency for financial support and Laboratoire de RMN et de Modelisation Moléculaire, ULP, Strasbourg, France, for facilities provided. Special gratitude is extended to R. Graff for technical assistance with the NMR measurements.

\section{References}

1. Mahato, S.B.; Nandy, A.K.; Roy, G. Phytochemistry 1992, 31, 2199.

2.Loganathan, D.; Trivedi, G.K.; Chary, K.V.R. Magn. Reson. Chem. 1990 28, 925.
3. Oliveira, E.F. In Contribuição ao Conhecimento Quimico de Plantas do Nordeste: Mentha villosa (Labiatae); Thesis, Universidade Federal do Ceará, Fortaleza, Brazil, 1995.

4. Matos, F.J.A. In Farmácias Vivas, 2ed. MV. e Atual, Universidade Federal do Ceará, Fortaleza, Brazil, 1991.

5. Muniz, M. Ciência Hoje 1990, 16, 68.

6. Reynolds, W.F.; Poplawski, P.; Enriquez, R.G.; Escobar, L.I.; Leon, I. Tetrahedron 1996, 42, 3419.

7. Faure, R.; Gaydou, E.M.; Wollenweber, E. J. Nat. Prod. 1991, 54, 1564.

8. Monte, F.J.Q.; Kintzinger, J.P.; Braz-Filho, R. Magn. Reson. Chem. 1997, 35, 802.

9. Schöder, H.; Haslinger, E. Magn. Reson. Chem. 1994, $32,12$.

10. Jimeno, M.J.; Rumbero, A.; Vázquez, P. Magn. Reson. Chem. 1995, 33, 408. 